\title{
Frequency-Domain Robust Performance Condition for Controller Uncertainty in SISO LTI Systems: A Geometric Approach
}

\author{
Vahid Raissi Dehkordi and Benoit Boulet \\ Department of Electrical and Computer Engineering, Centre for Intelligent Machines, McGill University, 633-3480 University Street, \\ Montréal, QC, Canada H3A $2 A 7$ \\ Correspondence should be addressed to Vahid Raissi Dehkordi, vahid@cim.mcgill.ca
}

Received 3 September 2008; Accepted 3 April 2009

Recommended by Andrea Garulli

This paper deals with the robust performance problem of a linear time-invariant control system in the presence of robust controller uncertainty. Assuming that plant uncertainty is modeled as an additive perturbation, a geometrical approach is followed in order to find a necessary and sufficient condition for robust performance in the form of a bound on the magnitude of controller uncertainty. This frequency domain bound is derived by converting the problem into an optimization problem, whose solution is shown to be more time-efficient than a conventional structured singular value calculation. The bound on controller uncertainty can be used in controller order reduction and implementation problems.

Copyright (c) 2009 V. Raissi Dehkordi and B. Boulet. This is an open access article distributed under the Creative Commons Attribution License, which permits unrestricted use, distribution, and reproduction in any medium, provided the original work is properly cited.

\section{Introduction}

In feedback control applications, the plant to be controlled is often modeled as a fixed linear time-invariant system. The modeling process typically neglects some of the plant's dynamics which results in model uncertainties. In spite of such differences between the plant and its corresponding nominal model, the designed controller must be able to control the plant, that is, it should guarantee robust stability under the new circumstances caused by the plant uncertainties [1].

Often, stability is not the only property of a closed-loop system that must be robust to plant perturbations. Tracking or regulation errors are caused by exogenous disturbances acting on the system, and plant perturbations can cause these errors to increase greatly. In such cases, the closed-loop performance can become unacceptable. Hence, it is necessary to check for both robust stability and robust performance of the system [2].

Based on the above observations, a robust controller is often designed and implemented in order to maintain the robust stability and performance of the system. But the controller itself may also have perturbations caused by a nonideal implementation, for example, discretization, order reduction, fixed point implementation of the controller, or environmental conditions such as temperature changes that can affect an analog controller. Therefore, it is reasonable to assume that the controller has uncertainty too $[3,4]$. Examples are found in real-time applications where the controller has to be retuned or redesigned several times due to drastic changes in system components $[5,6]$.

Some control design techniques result in having a finite impulse response (FIR) controller which is easy to implement [5]. On the other hand, classical controller synthesis algorithms such as DK iteration yield infinite impulse response (IIR) controllers $[7,8]$ which are not easily tuneable like FIR controllers can be. Thus, an implementation of an IIR controller should be robust to perturbations in the coefficients of the transfer function. Fast Fourier transform (FFT) techniques may also be used to design and implement the controller in the frequency domain [5]. The difference between the frequency response of the FFT-based controller and the desired frequency response can be represented by controller uncertainty [9].

Previous work using the gap metric takes both plant and controller uncertainties into account, explaining the trade-off between the uncertainties in order to maintain robust stability. This is done by providing upper bounds on 
admissible uncertainties which preserve closed-loop stability and a specified small tolerance on the input-output behavior of a feedback system $[10,11]$. In this work, however, the focus is solely on dealing with controller uncertainty based on apriori knowledge about the uncertain plant and required performance characteristics. In other words, there is no trade-off considered among the system uncertainties.

The presence of controller uncertainty in the closed-loop system requires a revision of robust stability and performance conditions. In the following problem formulation, the nominal plant and controller are given, and plant perturbation is modeled as additive uncertainty. Assuming that the controller perturbation is also modeled as an additive uncertainty, a frequency domain bound on the magnitude of the controller's uncertainty is derived. This bound provides information on how large a perturbation the controller can handle and, for real-time implementations, when to redesign the controller. In other words, it acts as a necessary and sufficient condition for maintaining robust performance, comparing to a previously derived sufficient condition [12]. In the case of alternate controller implementation methods, this bound provides information about how much deviation between the designed controller and its implementation is acceptable.

This article is organized as follows. Section 1 provides general information about the control loop structure and the robust performance problem. It discusses the results derived so far regarding a sufficient condition for maintaining robust performance. The main geometric approach is discussed in Section 2 which leads to the main theorem providing an optimization scheme in order to find a necessary and sufficient condition for robust performance in the form of an upper bound on the magnitude of the controller uncertainty. Finally, a detailed example is given in Section 3 as well as calculation time comparison between the proposed algorithm and bound calculation using conventional structured singular value calculation tools, followed by the conclusions.

\section{Problem Formulation}

A typical control loop is depicted in Figure 1, where the continuous-time, single-input, single-output, real-rational LTI systems $K_{0}(s)$ and $P_{0}(s)$ are the nominal controller and plant, respectively. Assuming that the nominal feedback control system is stable guarantees that all zeros of $1+$ $K_{0}(s) P_{0}(s)$ are located in the left half-plane [13]. In the perturbed case, the uncertainties are considered to be additive, that is, $P(s)=P_{0}(s)+\Delta_{P}(s)$ in which $\Delta_{P}(s)$ is characterized as the combination of a weighting function and a normalized stable uncertainty $\widetilde{\Delta}_{P}(s)[14]$ :

$$
\begin{gathered}
\Delta_{P}(s)=W_{P}(s) \widetilde{\Delta}_{P}(s), \quad \tilde{\Delta}_{P}(s) \in \mathscr{H}_{\infty}, \\
\left\|\tilde{\Delta}_{P}(s)\right\|_{\infty}<1 .
\end{gathered}
$$

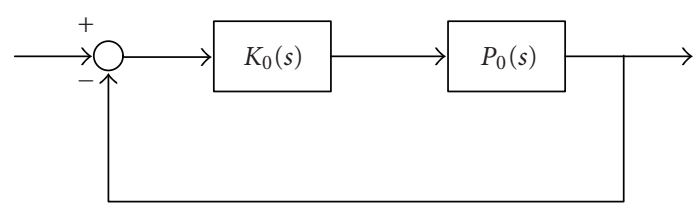

Figure 1: Typical control loop with nominal controller and plant.

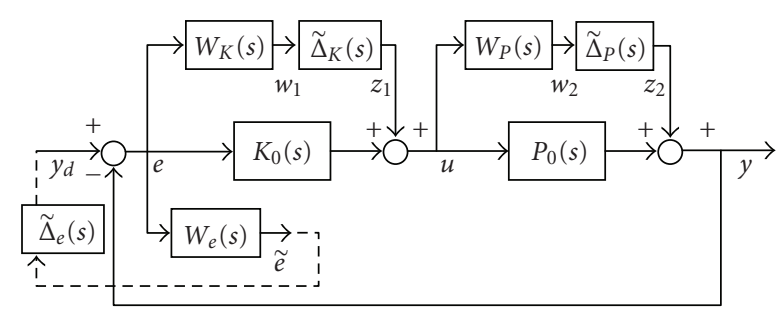

FIGURE 2: Expanded control loop with additive uncertainties and fictitious performance connection.

The controller perturbation is also modeled as a stable additive uncertainty, that is, $K(s)=K_{0}(s)+\Delta_{K}(s)$ where

$$
\begin{gathered}
\Delta_{K}(s)=W_{K}(s) \widetilde{\Delta}_{K}(s), \quad \tilde{\Delta}_{K}(s) \in \mathcal{H}_{\infty}, \\
\left\|\tilde{\Delta}_{K}(s)\right\|_{\infty}<1 .
\end{gathered}
$$

Despite being potentially conservative, an additive uncertainty can be well suited to model the effect of various sources of uncertainty in the plant and controller such as neglected dynamics and parametric perturbations. The control loop in Figure 1 is shown with controller and plant uncertainty in Figure 2.

The block $\widetilde{\Delta}_{e}(s)$ is a fictitious uncertainty introduced as a means of enforcing robust performance and appears only in the design stage

$$
\begin{gathered}
\Delta_{e}(s)=W_{e}(s) \tilde{\Delta}_{e}(s), \quad \tilde{\Delta}_{e}(s) \in \mathcal{H}_{\infty}, \\
\left\|\tilde{\Delta}_{e}(s)\right\|_{\infty}<1 .
\end{gathered}
$$

The uncertainty block $\widetilde{\Delta}_{e}(s)$ in conjunction with the weighting function $W_{e}(s)$ is included in the design in order to ensure that the error signal $e$ is held within acceptable limits. The system is said to have robust performance if $\left\|\left(y_{d} \mapsto e\right)(s)\right\|_{\infty} \leq 1$ for all admissible plant and controller perturbations $\left\|\tilde{\Delta}_{P}(s)\right\|_{\infty}<1,\left\|\tilde{\Delta}_{K}(s)\right\|_{\infty}<1$.

Lemma 1. Let $P_{0}, K_{0}, \widetilde{\Delta}_{P}, \widetilde{\Delta}_{K}$, and $W_{e}$ be samples of their respective transfer functions at frequency $\omega_{0}$, that is, $P_{0}=$ $\left.P_{0}(s)\right|_{s=j \omega_{0}}, \omega_{0} \in \mathbb{R}^{+}$. The system in Figure 2 has robust performance if and only if the following inequality holds at all frequencies $\omega_{0} \in \mathbb{R}^{+}$:

$$
\sup _{\substack{\left|\tilde{\Delta}_{K}\right|<1 \\\left|\tilde{\Delta}_{P}\right|<1}}\left(\left|\frac{W_{e}}{1+\left(P_{0}+W_{P} \tilde{\Delta}_{P}\right)\left(K_{0}+W_{K} \tilde{\Delta}_{K}\right)}\right|\right) \leq 1 .
$$




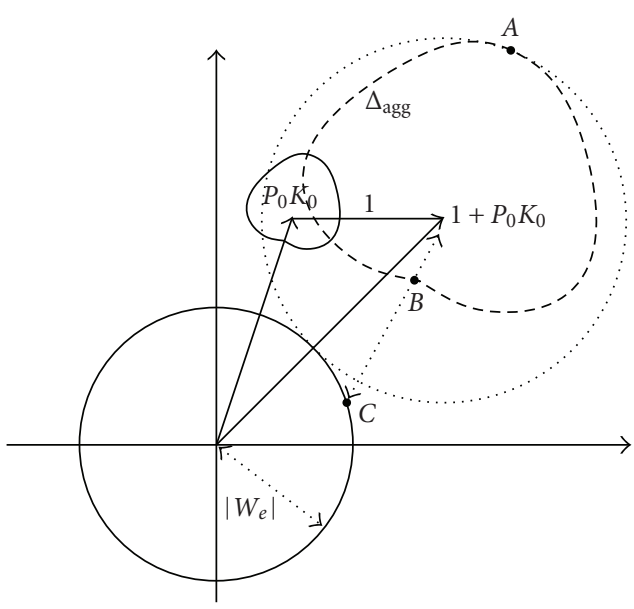

Figure 3: Nominal system characteristics, arbitrary aggregated uncertainty set and the sufficient upper bound.

The proof [12] consists of rearranging the whole system into an M- $\Delta$ interconnection [15] and then applying the main-loop theorem $[7,14]$ to the system.

The controller implementation problem is posed as follows. What is the maximum range of variation for the controller's additive uncertainty representing the discrepancy between the designed controller and its implemented version, as given by an upper bound for the uncertainty weighting function $W_{K}(s)$ ? The following lemma provides a sufficient condition for maintaining system robust performance in such a case.

Lemma 2. Assume that $W_{K}$ represents a sample of $W_{K}(s)$ at frequency $\omega_{0}$, that is, $W_{K}=\left.W_{K}(s)\right|_{s=j \omega_{0}}, \omega_{0} \in \mathbb{R}^{+}$. The system in Figure 2 has robust performance if the weighting function $W_{K}(s)$ bounding controller uncertainty satisfies the following inequality for all $\omega_{0} \in \mathbb{R}^{+}$:

$$
\left|W_{K}\right| \leq \frac{\left|1+P_{0} K_{0}\right|-\left|W_{P} K_{0}\right|-\left|W_{e}\right|}{\left|P_{0}\right|+\left|W_{P}\right|} .
$$

Proof. Since the normalized uncertainties appear in conjunction with their respective weighting functions in (1) and (2), the phase of the weighting functions can be absorbed into the normalized uncertainties remembering the fact that normalized uncertainties can have any phase angle. Rewriting the result of Lemma 1 leads to

$$
\begin{aligned}
& \left|\left(1+P_{0} K_{0}+K_{0}\left|W_{P}\right| \tilde{\Delta}_{P}+P_{0}\left|W_{K}\right| \tilde{\Delta}_{K}+\left|W_{P}\right|\left|W_{K}\right| \tilde{\Delta}_{P} \tilde{\Delta}_{K}\right)\right| \\
& \quad \geq\left|W_{e}\right|, \quad \forall\left|\tilde{\Delta}_{P}\right|<1, \forall\left|\widetilde{\Delta}_{K}\right|<1 .
\end{aligned}
$$

In the nominal case, $\left|1+P_{0} K_{0}\right| \geq\left|W_{e}\right|$. Therefore, the above inequality holds if and only if

$$
\begin{aligned}
& \left|\left(K_{0}\left|W_{P}\right| \tilde{\Delta}_{P}+P_{0}\left|W_{K}\right| \tilde{\Delta}_{K}+\left|W_{P}\right|\left|W_{K}\right| \tilde{\Delta}_{P} \tilde{\Delta}_{K}\right)\right| \\
& \quad \leq\left|1+P_{0} K_{0}\right|-\left|W_{e}\right|, \quad \forall\left|\widetilde{\Delta}_{P}\right|<1, \quad \forall\left|\tilde{\Delta}_{K}\right|<1 .
\end{aligned}
$$

The left-hand side of (7) contains all uncertain terms, therefore forming an aggregated complex uncertainty defined as

$$
\Delta_{\mathrm{agg}}:=K_{0}\left|W_{P}\right| \tilde{\Delta}_{P}+P_{0}\left|W_{K}\right| \tilde{\Delta}_{K}+\left|W_{P}\right|\left|W_{K}\right| \tilde{\Delta}_{P} \tilde{\Delta}_{K} .
$$

Back to (7) and using (8), $\left|\Delta_{\mathrm{agg}}\right|$ reaches its maximum, that is, the worst case scenario happens, when all of its three components are aligned along the same direction, forming a straight line in the complex plane. This happens if $\measuredangle \widetilde{\Delta}_{P}=$ $\measuredangle P_{0}$ and $\measuredangle \tilde{\Delta}_{K}=\measuredangle K_{0}$. Applying the phase constraints leads to (5) and completes the proof.

\section{Geometric Approach}

The bound in (5) as the result of Lemma 2, however, fails to provide a necessary and sufficient condition for robust performance. This is because of the fact that the upper bound calculation takes into account the maximum possible magnitude of $\Delta_{\text {agg }}$ which occurs at a certain angle, that is, $\measuredangle P_{0} K_{0}$, and not the maximum magnitude at every angle. Keeping in mind that $P_{0}$ and $K_{0}$ are samples of their respective transfer functions at a certain frequency, Figure 3 shows the difference between the measurement in computing the sufficient upper bound and the actual measurements which should be done in order to find an exact bound. Note that $\Delta_{\mathrm{agg}}$ is shown as some arbitrary shape.

The dashed contour bounds all possible occurrences of $\Delta_{\text {agg, }}$, centered at $1+P_{0} K_{0}$ as in (6) where it appears in conjunction with the aggregated uncertainty set spanned by all admissible $\Delta_{\text {agg. }}$. Point $A$ shows the location at which the maximum magnitude of $\Delta_{\text {agg }}$ occurs, and the dotted circle centered at $1+P_{0} K_{0}$ passing through $A$ represents the upper bound calculated in (5). The circle touches the solid circle of radius $\left|W_{e}\right|$, maintaining the inequalities (6) or (7). Point $B$, however, as a typical boundary point of $\Delta_{\text {agg }}$ facing the solid circle, is still far from graphically touching the solid circle which means that there is still room for $\left|W_{K}\right|$ to increase and subsequent expansion of $\Delta_{\mathrm{agg}}$ around $1+P_{0} K_{0}$, in this case until it reaches point $C$.

Therefore, in order to find a necessary and sufficient condition, the magnitude of the aggregated uncertainty set should be verified for a variety of border locations and not just at the peak point A. In fact, the maximum magnitude calculation is only necessary for a certain area facing the solid circle of radius $\left|W_{e}\right|$. This is illustrated in Figure 4(a), and the following lemma narrows down the critical uncertainty range.

Lemma 3. The robust performance of the system is guaranteed if and only if for all $\theta \in\left[-\theta_{0}, \theta_{0}\right]$, where $\theta_{0}=\operatorname{Arcsin}\left(\mid W_{e} /(1+\right.$ $\left.\left.P_{0} K_{0}\right) \mid\right)$, the maximum magnitude of the aggregated uncertainty at the direction of $\theta$ is less than or equal to $x(\theta)$ defined as

$$
x(\theta)=\frac{\left|W_{e}\right| \sin \left[\operatorname{Arcsin}\left(\left|\left(\left(1+P_{0} K_{0}\right) / W_{e}\right) \sin \theta\right|\right)-|\theta|\right]}{|\sin \theta|} .
$$

Proof. Referring to Figure 4(a), it is clear that a violation of (6) can only happen within the indicated range for $\theta$. In order 
to find $x(\theta)$, the sine rule is used according to Figure 4(b). Knowing that $\varphi$ is an obtuse angle,

$$
\begin{gathered}
\frac{\left|W_{e}\right|}{|\sin \theta|}=\frac{\left|1+P_{0} K_{0}\right|}{\sin \varphi}, \\
\varphi=\pi-\operatorname{Arcsin}\left(\left|\frac{1+P_{0} K_{0}}{W_{e}} \sin \theta\right|\right), \\
\theta_{x}=\operatorname{Arcsin}\left(\left|\frac{1+P_{0} K_{0}}{W_{e}} \sin \theta\right|\right)-|\theta| .
\end{gathered}
$$

Using the sine rule again leads to

$$
\frac{\left|W_{e}\right|}{|\sin \theta|}=\frac{x(\theta)}{\sin \theta_{x}}
$$

which with (10) completes the proof. Note that the angle $\theta$ appears as $|\theta|$ because of the negative values it can take within its corresponding range.

On the other hand, based on the definition of the uncertainty, it is preferable to do the calculations with respect to $P_{0} K_{0}$ rather than $1+P_{0} K_{0}$ as the latter is merely a shifted version of the former in a geometric sense. Therefore, the following angle conversions as shown in Figure 4(c) are applied:

$$
\theta_{P K}=\pi+\theta-\theta_{d}
$$

where

$$
\theta_{d}=\varangle\left(1+P_{0} K_{0}\right)-\varangle\left(P_{0} K_{0}\right)
$$

The aggregated uncertainty $\Delta_{\text {agg }}$ can be rewritten as

$$
\begin{aligned}
\Delta_{\mathrm{agg}} & =K_{0}\left|W_{P}\right| \tilde{\Delta}_{P}+P_{0}\left|W_{K}\right| \tilde{\Delta}_{K}+\left|W_{P}\right|\left|W_{K}\right| \tilde{\Delta}_{P} \tilde{\Delta}_{K} \\
& =P_{0} K_{0}\left(\frac{\left|W_{P}\right|}{P_{0}} \tilde{\Delta}_{P}+\frac{\left|W_{K}\right|}{K_{0}} \tilde{\Delta}_{K}+\frac{\left|W_{P}\right|\left|W_{K}\right|}{P_{0} K_{0}} \tilde{\Delta}_{P} \widetilde{\Delta}_{K}\right) \\
& =P_{0} K_{0}\left(\left|\frac{W_{P}}{P_{0}}\right| \tilde{\Delta}_{P}+\left|\frac{W_{K}}{K_{0}}\right| \tilde{\Delta}_{K}+\left|\frac{W_{P}}{P_{0}}\right| \cdot\left|\frac{W_{K}}{K_{0}}\right| \tilde{\Delta}_{P} \tilde{\Delta}_{K}\right) \\
& \triangleq P_{0} K_{0}\left(r_{P} \tilde{\Delta}_{P}+r_{K} \tilde{\Delta}_{K}+r_{P} r_{K} \tilde{\Delta}_{P} \tilde{\Delta}_{K}\right)
\end{aligned}
$$

where the real positive variables $r_{P}$ and $r_{K}$ in (14) stand for the multiplicative uncertainty bounds of the plant and controller. Note that the phases of $P_{0}$ and $K_{0}$ appearing in the denominators are absorbed into the normalized uncertainties in a similar way to the proof of Lemma 1. The variable $r_{P}$ is known, while the variable $r_{K}$ should be maximized in order to provide the controller with a maximum possible uncertainty level without losing robust performance. Therefore, the problem of finding a necessary and sufficient robust performance condition as an upper bound for $\left|W_{K}\right|$ is transformed into finding the maximum possible value for $r_{K}$.

Changing the geometric coordinates by normalizing (14) by $P_{0} K_{0}$, the problem is simplified as shown in Figure $4(d)$. Now the main result is expressed in the following theorem.
Theorem 1. Under the assumptions given in Lemmas 1 and 2 and based on definitions given in (14), the largest multiplicative controller uncertainty level $r_{K \max }$ maintaining robust performance of the system is given at each frequency by:

$$
\begin{aligned}
& r_{K \max } \\
& =\min _{\theta_{P K} \in \Omega} \underset{r_{K} \in \mathbb{R}_{+}}{\operatorname{argmin}}\left(\left|\frac{x\left(\theta_{P K}\right)}{\left|P_{0} K_{0}\right|}-\max _{\substack{\omega_{1} \\
\omega_{2}}} f\left(\omega_{1}, \omega_{2}, r_{P}, r_{K}, \theta_{P K}\right)\right|\right),
\end{aligned}
$$

$$
\Omega \triangleq\left[\pi-\theta_{0}-\theta_{d}, \pi+\theta_{0}-\theta_{d}\right]
$$

where using (9) and (12), $x\left(\theta_{P K}\right)$ is found as

$$
x\left(\theta_{P K}\right)=\frac{\left|W_{e}\right| \sin [\mathcal{A}]}{\left|\sin \left(\theta_{P K}+\theta_{d}\right)\right|},
$$

where $\mathcal{A} \triangleq \operatorname{Arcsin}\left(\left|\left((1+P K) / W_{e}\right) \sin \left(\theta_{P K}+\theta_{d}\right)\right|\right)-\mid \theta_{P K}+$ $\theta_{d}-\pi \mid$ and function $f$ is given as

$$
\begin{aligned}
f\left(\omega_{1}, \omega_{2}, r_{P}, r_{K}, \theta_{P K}\right) \triangleq & r_{P} \cos \left(\omega_{1}\right)+r_{K} \cos \left(\omega_{2}\right) \\
& +r_{P} r_{K} \cos \left(\omega_{1}+\omega_{2}+\theta_{P K}\right) \\
= & r_{P} \cos \left(\omega_{a}-\theta_{P K}\right)+r_{K} \cos \left(\omega_{b}-\theta_{P K}\right) \\
& +r_{P} r_{K} \cos \left(\omega_{a}+\omega_{b}-\theta_{P K}\right),
\end{aligned}
$$

where $\omega_{a}=\measuredangle \tilde{\Delta}_{P}, \omega_{b}=\measuredangle \widetilde{\Delta}_{K}, \omega_{1}=\omega_{a}-\theta_{P K}, \omega_{2}=\omega_{b}-\theta_{P K}$, and $r_{P}$ is a constant.

Proof. The innermost optimization in (15) is based on the function $f$ which gives the magnitude of $r_{P} \widetilde{\Delta}_{P}+r_{K} \widetilde{\Delta}_{K}+$ $r_{P} r_{K} \tilde{\Delta}_{P} \widetilde{\Delta}_{K}$ at a given direction $\theta_{P K}$. In order to find the maximizing parameters, the gradient is found and set equal to zero [16]:

$$
\begin{aligned}
& \frac{\partial f}{\partial \omega_{1}}=-r_{P} \sin \left(\omega_{1}\right)-r_{P} r_{K} \sin \left(\omega_{1}+\omega_{2}+\theta_{P K}\right)=0, \\
& \frac{\partial f}{\partial \omega_{2}}=-r_{K} \sin \left(\omega_{2}\right)-r_{P} r_{K} \sin \left(\omega_{1}+\omega_{2}+\theta_{P K}\right)=0,
\end{aligned}
$$

which leads to the following equations:

$$
\begin{gathered}
r_{P} \sin \left(\omega_{1}\right)=r_{K} \sin \left(\omega_{2}\right), \\
\sin \left(\omega_{1}\right)=-r_{K} \sin \left(\omega_{1}+\omega_{2}+\theta_{P K}\right), \\
\sin \left(\omega_{2}\right)=-r_{P} \sin \left(\omega_{1}+\omega_{2}+\theta_{P K}\right) .
\end{gathered}
$$

From (19), the following two closed-form equations are obtained with respect to $\omega_{1}$ and $\omega_{2}$ :

$$
\begin{aligned}
& \frac{\sin ^{2}\left(\omega_{1}\right)}{r_{K}^{2}}=\frac{\sin ^{2}\left(\omega_{1}+\theta_{P K}\right)}{\left(1+r_{P}^{2}\right)+2 r_{P} \cos \left(\omega_{1}+\theta_{P K}\right)}, \\
& \frac{\sin ^{2}\left(\omega_{2}\right)}{r_{P}^{2}}=\frac{\sin ^{2}\left(\omega_{2}+\theta_{P K}\right)}{\left(1+r_{K}^{2}\right)+2 r_{K} \cos \left(\omega_{2}+\theta_{P K}\right)} .
\end{aligned}
$$


After finding the solutions for either $\omega_{1}$ or $\omega_{2}$ and then using (20) and (18), the local maxima are found, and $\max _{\omega_{1}, \omega_{2}} f\left(\omega_{1}, \omega_{2}, r_{P}, r_{K}, \theta_{P K}\right)$ will be equal to the largest local maximum. Note that the phase of $W_{K}$ is never taken into account, and the bound is given for its magnitude.

The intermediate search level is done with respect to $r_{K}$ in (15), which returns the maximum possible magnitude of $r_{K}$ or the controller multiplicative uncertainty. This particular value of $r_{K}$ is associated with $\theta_{P K}$.

The outermost search level is performed on all possible $\theta_{P K}$ within the set $\Omega$, that is, for every $\theta_{P K}$ the maximum acceptable $r_{K}$ is calculated. Then, the final upper bound on $\left|W_{K}\right|$ is given by the minimum $r_{K \max }$ multiplied by $\left|K_{0}\right|$, as it ensures that at all critical angles the robust performance is guaranteed while providing maximum possible freedom for $\left|W_{K}\right|$.

Remarks 1. Regarding the innermost search level in (15), no analytic solution could be found for $\omega_{1}$ and $\omega_{2}$ using (21) and (22). Therefore, a numerical search is applied in order to find the solutions, which is presented in Proposition 1. In the intermediate search level, the initial value of $r_{K}$ is set as the upper bound previously found as (5) scaled by $\left|K_{0}\right|$. This makes sure that the robust performance condition is not violated at the beginning or in other words, $\max _{\omega_{1}, \omega_{2}} f\left(\omega_{1}, \omega_{2}, r_{P}, r_{K}, \theta_{P K}\right)$ is initially less than $x\left(\theta_{P K}\right) /\left|P_{0} K_{0}\right|$, and the search on $r_{K}$ stops when the boundary is reached. Also regarding the outermost search level, the search on $\theta_{P K}$ can be narrowed down to a limited range, which is covered in Proposition 2.

The optimization routine in

$$
\begin{aligned}
& r_{K \max } \\
& =\min _{\theta_{P K} \in \Omega} \underset{r_{K} \in \mathbb{R}_{+}}{\operatorname{argmin}}\left(\left|\frac{x\left(\theta_{P K}\right)}{\left|P_{0} K_{0}\right|}-\max _{\omega_{1}} f\left(\omega_{1}, \omega_{2}, r_{P}, r_{K}, \theta_{P K}\right)\right|\right)
\end{aligned}
$$

(15) is implemented using functions included in the MATLAB optimization toolbox. The innermost and intermediate search levels employ the fzero function while the outermost level uses fminbnd. The function fzero is based on golden section search and parabolic interpolation while fminbnd uses bisection and interpolation which also works in case of nondifferentiable minimization scenarios.

Proposition 1. The numerical search required in order to find the solution for (21) and (22) is simplified by narrowing down the search region for $\omega_{1}$ to

$$
\begin{aligned}
\omega_{1} & \in\left[-\pi, \min \left\{-\pi+\arcsin \left(\frac{r_{K}}{r_{P}}\right),-\theta_{P K}\right\}\right] \\
\cup & {\left[\max \left\{-\arcsin \left(\frac{r_{K}}{r_{P}}\right),-\theta_{P K}\right\}, 0\right] } \\
\cup & {\left[0, \min \left\{\arcsin \left(\frac{r_{K}}{r_{P}}\right), \pi-\theta_{P K}\right\}\right] } \\
\cup & {\left[\max \left\{\pi-\arcsin \left(\frac{r_{K}}{r_{P}}\right), \pi-\theta_{P K}\right\}, \pi\right] }
\end{aligned}
$$

for the case $r_{K}<r_{P}$ and narrowing down the search region for $\omega_{2}$ to

$$
\begin{aligned}
\omega_{2} & \in\left[-\pi, \min \left\{-\pi+\arcsin \left(\frac{r_{P}}{r_{K}}\right),-\theta_{P K}\right\}\right] \\
& \cup\left[\max \left\{-\arcsin \left(\frac{r_{P}}{r_{K}}\right),-\theta_{P K}\right\}, 0\right] \\
& \cup\left[0, \min \left\{\arcsin \left(\frac{r_{P}}{r_{K}}\right), \pi-\theta_{P K}\right\}\right] \\
& \cup\left[\max \left\{\pi-\arcsin \left(\frac{r_{P}}{r_{K}}\right), \pi-\theta_{P K}\right\}, \pi\right]
\end{aligned}
$$

for the case $r_{K}>r_{P}$.

The proof is given in the appendix.

Proposition 2. Considering the definitions given in Theorem 1, the following equality holds for all values of $r_{P}, r_{K}$ and $\theta_{P K}$ :

$$
\max _{\substack{\omega_{1} \\ \omega_{2}}} f\left(\omega_{1}, \omega_{2}, r_{P}, r_{K}, \theta_{P K}\right)=\max _{\substack{\omega_{1} \\ \omega_{2}}} f\left(\omega_{1}, \omega_{2}, r_{P}, r_{K},-\theta_{P K}\right) .
$$

As a result, the search on $\theta_{P K}$ can be restricted to the following intervals:

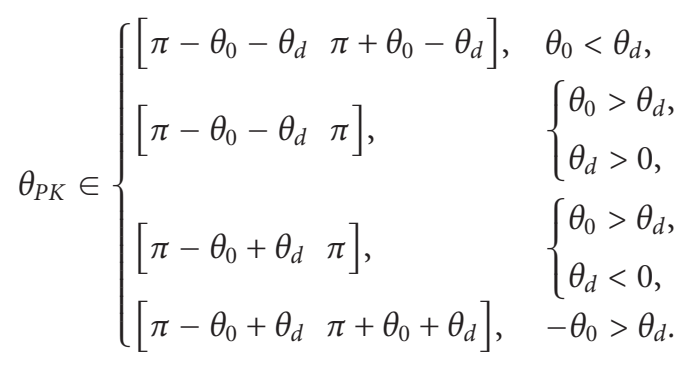

The proof is given in the appendix.

\section{Example}

Consider the following system setup:

$$
\begin{aligned}
& P_{0}(s)=\frac{10}{s+2}, \\
& W_{P}(s)=\frac{2}{s+4}, \\
& W_{e}(s)=\frac{50}{s+3} .
\end{aligned}
$$

The optimal robust controller is derived as follows using DKiteration:

$$
\begin{aligned}
K_{0}(s)= & -2.8726 \times 10^{7} \\
& \times \frac{(s+1147)(s+4.741)(s+4.492)}{\left(s+6.461 \times 10^{4}\right)(s+1064)(s+11.51)(s+11.19)} \\
& \times \frac{(s+3.032)(s+2.253)(s+2)}{(s+5.033)(s+3)(s+2.39)} .
\end{aligned}
$$




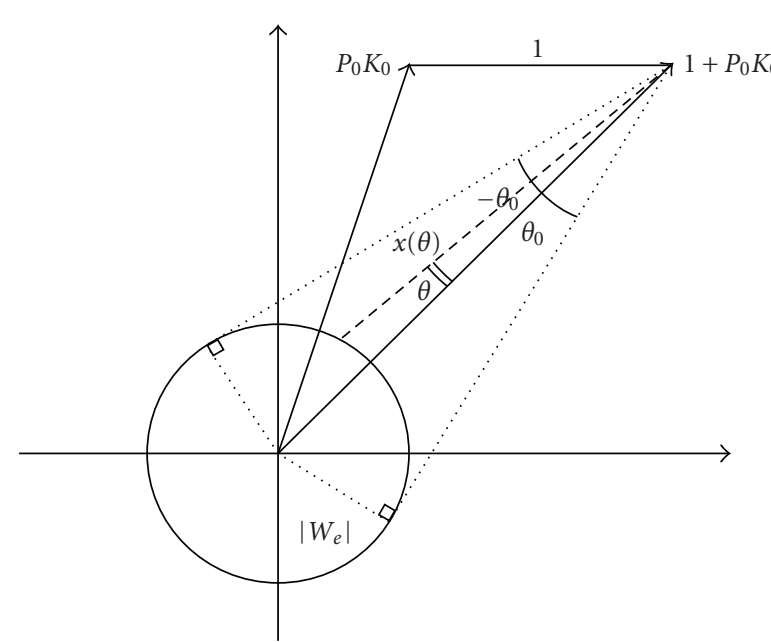

(a)

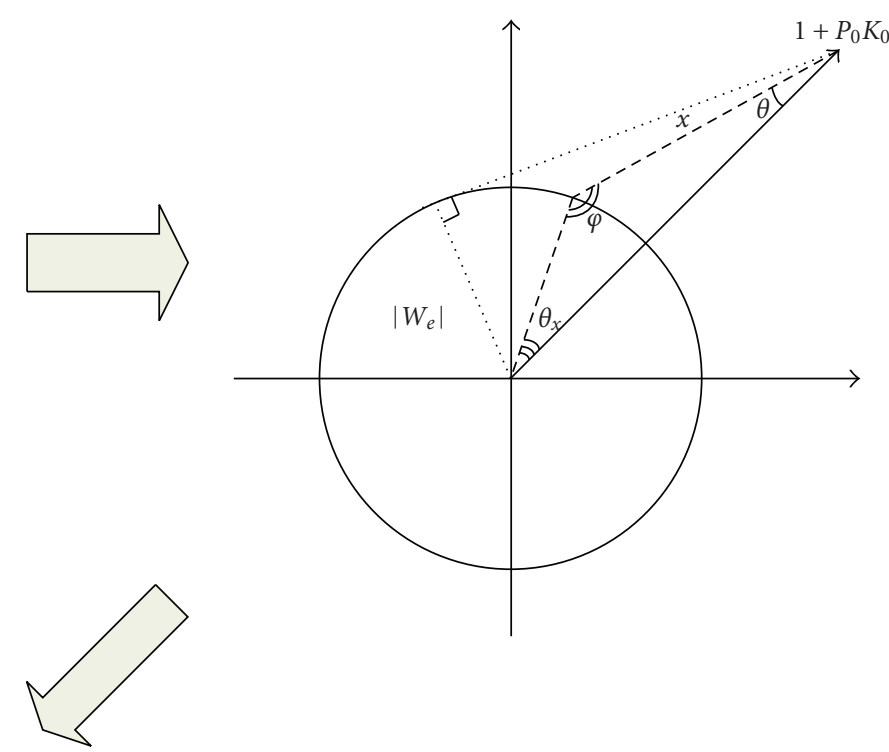

(b)

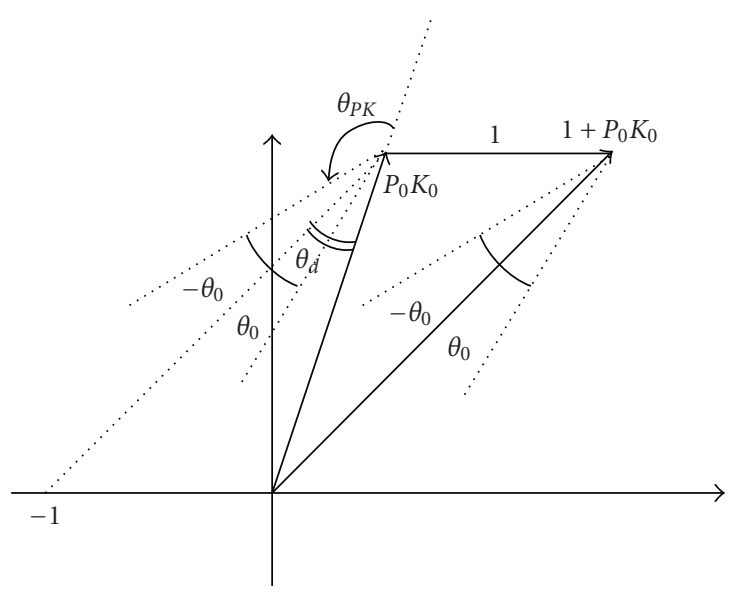

(c)

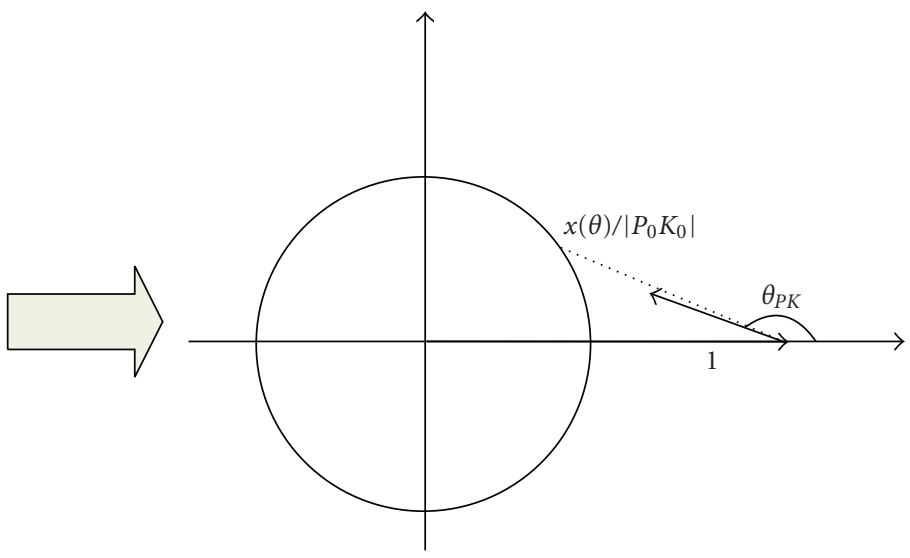

(d)

FIGURE 4: (a) The critical range for maximum possible magnitude verification, (b) limit calculation regarding $\theta$, (c) angle conversion, and (d) geometric transformation after normalization.

Assuming that the controller will suffer from having uncertainty modeled as an additive perturbation as in (2), it is desired to find an exact bound for $\left|W_{K}(j \omega)\right|$ as a necessary and sufficient condition for maintaining robust performance.

The calculation is performed over a set of 200 logarithmically equidistant points distributed over $\left[1 \mathrm{e}^{-2} \mathrm{~Hz}, 1 \mathrm{e}^{5} \mathrm{~Hz}\right]$ frequency span meaning that the search for optimum bound is performed 200 times. Both the sufficient upper bound given by (5) and the necessary and sufficient bound derived by solving (15) are shown in Figure 5.

The sufficient bound is obviously more conservative than the necessary and sufficient bound. In order to verify the accuracy of the necessary and sufficient bound, an eleventhorder weighting function is approximated in order to serve as $W_{K}$. The approximation is done in such a way that $\left|W_{K}\right|$ is always less than or equal to the bound. Then, the structured singular value of $M, \mu_{\Delta}(M)$, as in Lemma 1 is calculated in order to verify system's robust performance by comparing it to 1 [14]. Figure 6 shows the approximated weighting function and the corresponding $\mu_{\Delta}(M)$. As seen in Figure 6(b), $\mu_{\Delta}(M)$ is fairly close but less than 1 , and therefore, the robust performance is guaranteed. Also it can be seen that it almost follows the oscillations in $\left|W_{K}\right|$ such that a peak occurs in $\mu_{\Delta}(M)$ when $\left|W_{K}\right|$ gets close to the necessary and sufficient bound. 


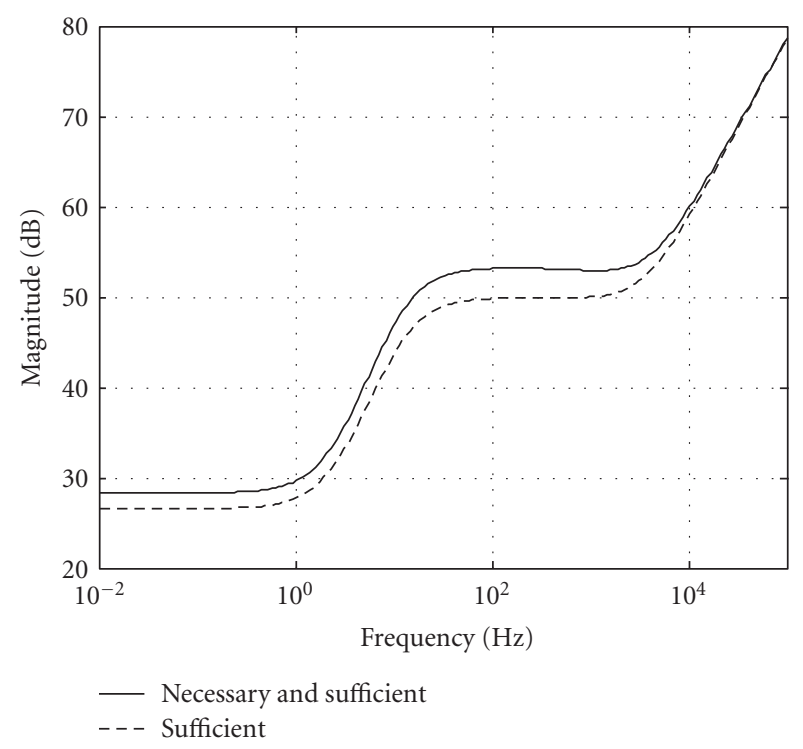

FIGURE 5: The robust performance bounds derived for the given system configuration.

TABLE 1: The average computation time needed for bound calculation.

\begin{tabular}{lcc}
\hline Method & Proposed method & $\mu$-based \\
\hline Time $(\mathrm{sec})$ & 88.9 & 434.8 \\
\hline
\end{tabular}

TABle 2: Partitioning of search domain of $\boldsymbol{\omega}_{1}$ in (21).

\begin{tabular}{lcc}
\hline$\omega_{1}(\mathrm{rad})$ & LHS & RHS \\
\hline$-\pi$ & 0 & + \\
$-\theta_{P K}$ & + & 0 \\
0 & 0 & + \\
$\pi-\theta_{P K}$ & + & 0 \\
$\pi$ & 0 & + \\
\hline
\end{tabular}

In order to verify the bound more closely, the same weighting function approximation and $\mu_{\Delta}(M)$ calculation process is repeated for a partial frequency range so that a smoother range of the bound is taken into account, leading to a more marginal case observation. Figure 7 (a) shows an accurate approximation which leads $\mu_{\Delta}(M)$ to be much closer to 1 comparing to the previous observation, this time almost higher than 0.98 as in Figure 7(b).

In order to compare the proposed method with conventionally used ones, a benchmark test is done in order to observe the time efficiency of our method. The reference method used in this comparison employs the structured singular value calculation directly, relying on well-developed $\mu_{\Delta}(M)$ calculation routines [7]. In order to find the necessary and sufficient robust performance bound, the following expression has to be evaluated at every frequency point:

$$
\arg \max _{r}\left[\max _{\theta} \mu_{\Delta}(M(r, \theta))\right] \leq 1,
$$

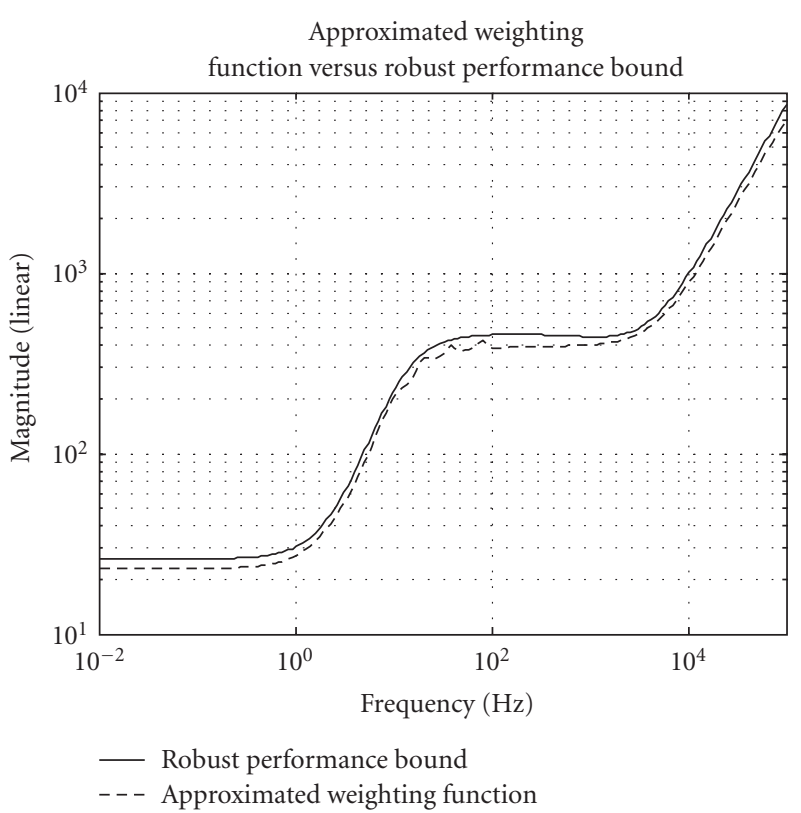

(a)

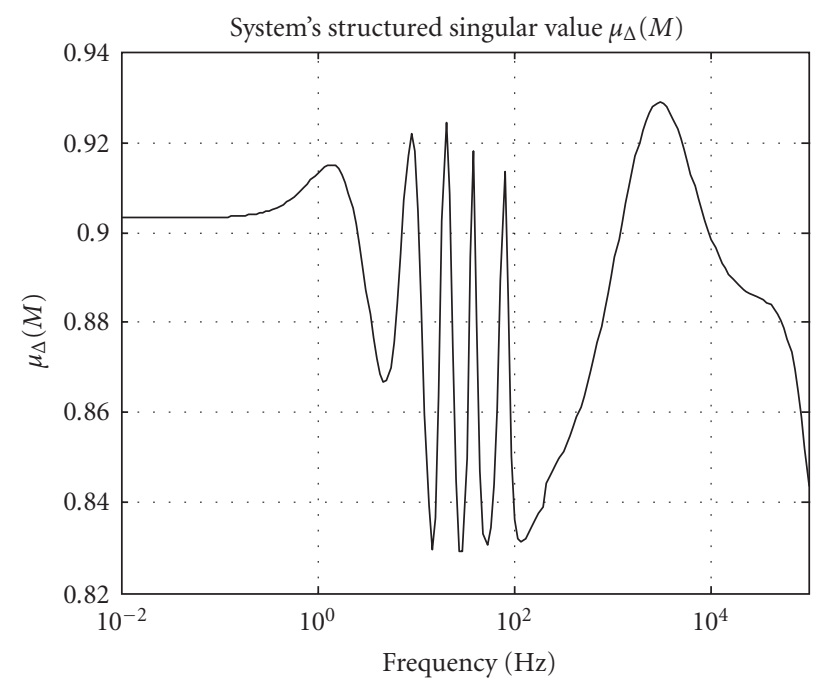

(b)

FIGURE 6: (a) The robust performance bound and the approximated weighting function $W_{K}$, (b) with its respective structured singular value plot.

where $-\pi \leq \theta<\pi$, and the matrix $M$ is the same matrix derived $M-\Delta$ in interconnection, thus including the term $W_{K}=r e^{i \theta}$. This search method starts with $r$ initially set equal to the sufficient robust stability bound as in (5), and verifies that all possible complex-valued combinations for $W_{K}$ keep $\mu_{\Delta}(M)$ less than 1 . The value of $r$ is increased until it happens that some combinations with this magnitude violate the robust performance condition. This final value will be the necessary and sufficient robust performance bound at a certain frequency point. Therefore, the upper bound is formed by repeating the same routine at each desired frequency point. This reference method also employs 


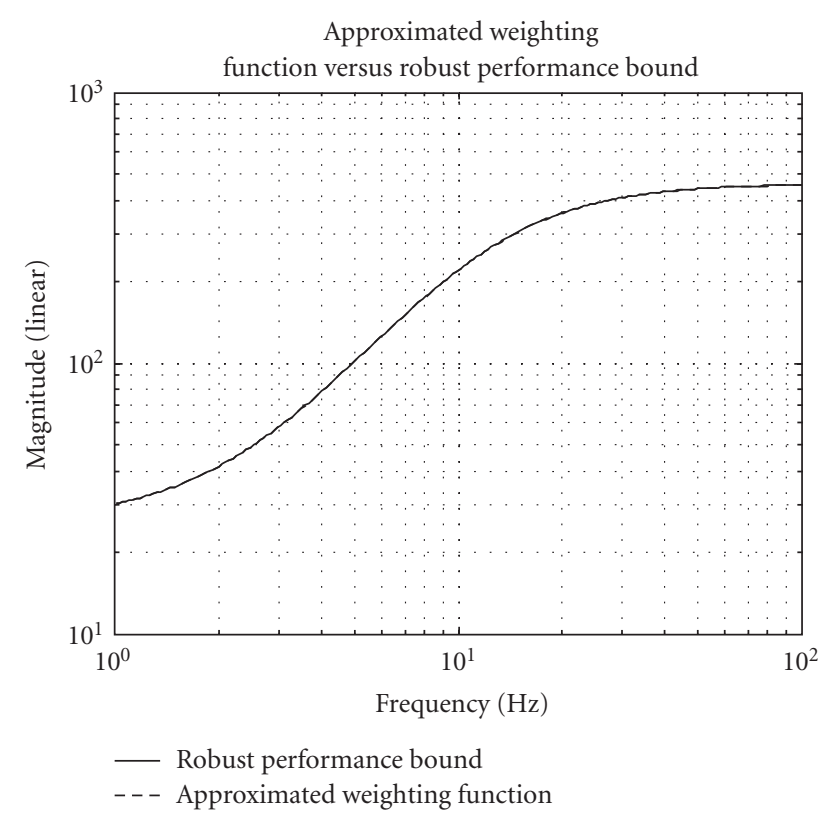

(a)

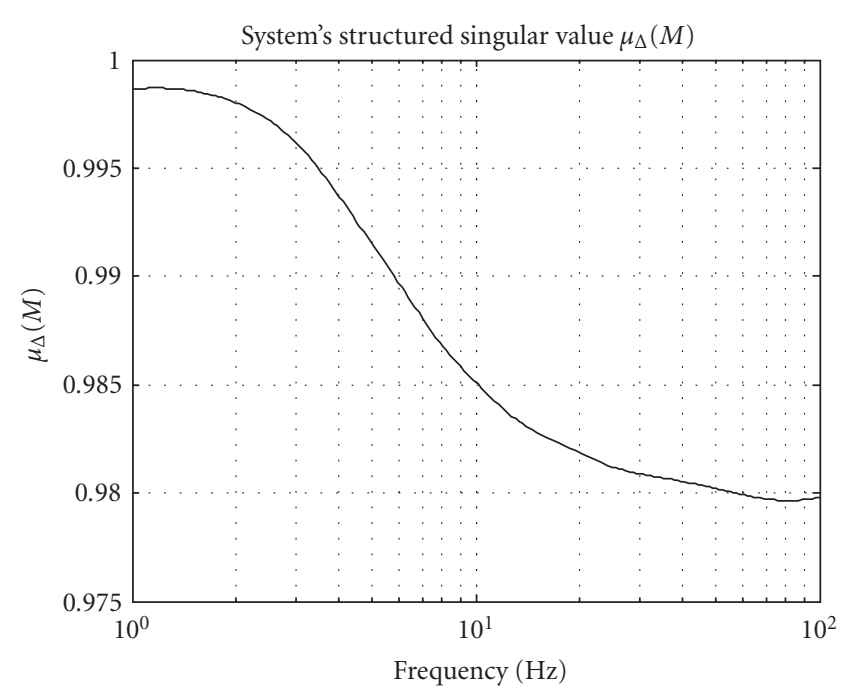

(b)

FIGURE 7: (a) The robust performance bound and the approximated weighting function $W_{K}$, (b) with its respective structured singular value plot for a limited frequency range.

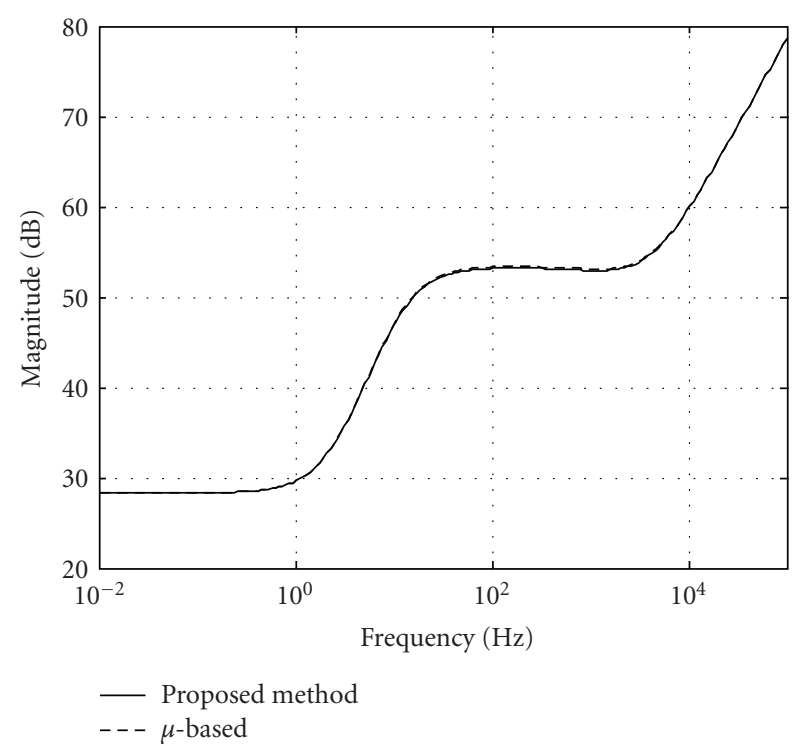

FIGURE 8: The necessary and sufficient robust performance bounds for controller uncertainty derived by the proposed method and using $\mu$-based calculation.

three search levels as $\mu_{\Delta}(M)$ calculation is considered to be a search routine, by definition.

With the same system configuration given at the beginning of the section, the above routine is run at the same frequency points fed through the proposed method, and the resulting controller uncertainty bound is shown in Figure 8 along with the bound shown in Figure 5. The derived bounds

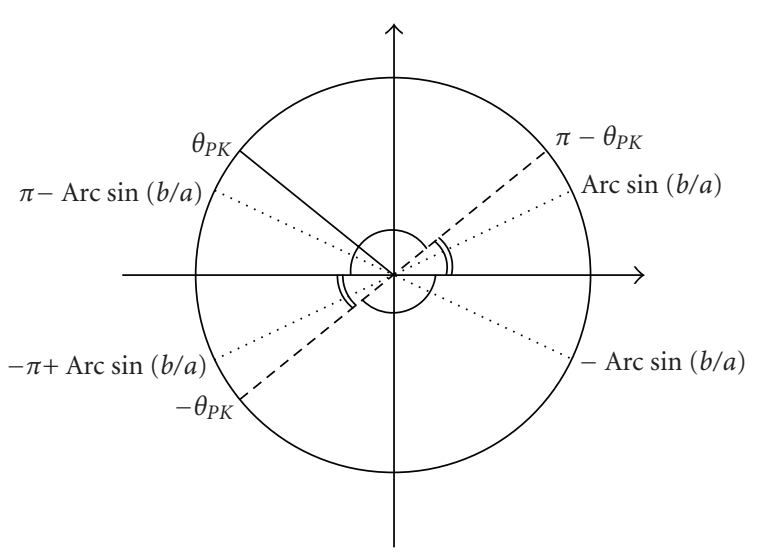

FIGURE 9: The angle boundaries versus primary search regions.

are very close which enforces the claimed accuracy of the proposed method. The average computation time for the above example over 10 executions using an Intel Pentium IV computer for both methods is tabulated in Table 1.

Clearly, the proposed method works 4-5 times faster than the $\mu$-based method.

\section{Conclusion}

In this article, a geometric approach is used in order to find a necessary and sufficient robust performance condition for a single-input single-output LTI system in the form of an upper bound on the magnitude of additive controller uncertainty. The bound is derived numerically using a three- 
level optimization which is made efficiently based on the propositions discussed about the problem formulation. The provided example demonstrates the accuracy of the proposed algorithm in determining the maximum possible size of controller uncertainty maintaining robust performance. The algorithm is shown to be more time-efficient when compared to the conventional structured singular value calculation tools under the same accuracy requirements.

\section{Appendix}

Proof of Proposition 1. For the case where $r_{\mathbf{K}}<r_{\mathbf{P}}$, (20) constrains $\omega_{1}$ as follows:

$$
\left|\sin \left(\omega_{1}\right)\right|<\frac{r_{\mathbf{K}}}{r_{\mathbf{P}}}<1 .
$$

At the same time, (21) should be solved for $\omega_{1}$. Since a closedform solution does not exist, all we can do is to restrict the search domain in order to speed up the solution process. Both sides of (21) are real and nonnegative. Observing the two sides leads to the following table assuming $0 \leq \theta_{\mathrm{PK}} \leq \pi$.

Therefore, the union of the following intervals should be searched in order to find the solutions to (21):

$$
\begin{aligned}
& \omega_{1} \in\left[-\pi,-\theta_{P K}\right] \\
& \cup\left[-\theta_{P K}, 0\right] \\
& \cup\left[0, \pi-\theta_{P K}\right] \\
& \cup\left[\pi-\theta_{P K}, \pi\right],
\end{aligned}
$$

as each region will have at least one solution. However, there is no need to search the entire unit circle as adding up the four regions suggests to. Using (A.1), the search region is constrained to (24). Figure 9 shows how the four search regions in (24) are derived.

For the case $r_{K}>r_{P},(20)$ constrains $\omega_{2}$ as follows:

$$
\left|\sin \left(\omega_{2}\right)\right|<\frac{r_{P}}{r_{K}}<1 .
$$

At the same time, (22) should be solved for $\omega_{2}$. With the same justification given for the above case and using (A.3), the final search region is given by (25) and the proof is completed.

Proof of Proposition 2. The equality $f\left(-\omega_{1},-\omega_{2}, r_{P}, r_{K}\right.$, $\left.-\theta_{P K}\right)=f\left(\omega_{1}, \omega_{2}, r_{P}, r_{K}, \theta_{P K}\right)$ holds for every value of $\omega_{1}, \omega_{2}$, and $\theta_{P K}$. Therefore, if the pair $\left(\omega_{1_{p}}, \omega_{2_{p}}\right)$ is the maximizing solution for $f\left(\omega_{1}, \omega_{2}, r_{P}, r_{K}, \theta_{P K}\right),\left(-\omega_{1_{p}},-\omega_{2_{p}}\right)$ will be the maximizing solution for $f\left(\omega_{1}, \omega_{2}, r_{P}, r_{K},-\theta_{P K}\right)$. Following the same argument, there will not be any other maximizing solution for $f\left(\omega_{1}, \omega_{2}, r_{P}, r_{K},-\theta_{P K}\right)$ because if such a solution exists, then its negative should also be the maximizing solution for $f\left(\omega_{1}, \omega_{2}, r_{P}, r_{K}, \theta_{P K}\right)$ which is a contradiction as $\left(\omega_{1_{p}}, \omega_{2_{p}}\right)$ is already found as the global maximizing solution. This symmetrical property can also be derived intuitively from Figure 4(d).

As a result, there is no need to search for the maximizing solution of $f\left(\omega_{1}, \omega_{2}, r_{P}, r_{K}, \theta_{P K}\right)$ in $-\pi \leq \theta_{P K} \leq 0$ because of the symmetry with respect to $\theta_{P K}$. Thus, regarding (16) the intervals in (27) are derived.

\section{References}

[1] C. A. Desoer and M. Vidyasagar, Feedback Systems: InputOutput Properties, Academic Press, New York, NY, USA, 1975.

[2] K. Zhou, J. C. Doyle, and K. Glover, Robust and Optimal Control, Prentice-Hall, Upper Saddle River, NJ, USA, 1996.

[3] G.-H. Yang, J. L. Wang, and C. Lin, " $\mathrm{H}_{\infty}$ control for linear systems with controller uncertainty," in Proceedings of the American Control Conference (ACC'99), vol. 5, pp. 3377-3381, San Diego, Calif, USA, June 1999.

[4] K. Zheng, A. H. Lee, J. Bentsman, and C. W. Taft, "Steadystate bumpless transfer under controller uncertainty using the state/output feedback topology," in Proceedings of the 43rd IEEE Conference on Decision and Control (CDC'04), vol. 2, pp. 1341-1346, Atlantis, Bahamas, December 2004.

[5] B. Boulet, Y. Duan, and H. Michalska, "An LMI approach to IMC-based robust tunable control," in Proceedings of the American Control Conference (ACC '03), vol. 1, pp. 821-826, Denver, Colo, USA, June 2003.

[6] A. Saengdeejing and Z. Qu, "Simplified robust control for nonlinear uncertain systems: a method of projection and online estimation," in Proceedings of the American Control Conference (ACC '02), vol. 5, pp. 3425-3430, Anchorage, Alaska, USA, May 2002.

[7] A. Packard and J. C. Doyle, "The complex structured singular value," Automatica, vol. 29, no. 1, pp. 71-109, 1993.

[8] G. J. Balas and J. C. Doyle, "Robust control of flexible modes in the controller crossover region," AIAA Journal of Guidance, Dynamics and Control, vol. 12, no. 2, pp. 370-377, 1994.

[9] V. R. Dehkordi, F. Labeau, and B. Boulet, "Piece-wise linear DFT interpolation for IIR systems: performance and error bound computation," in Proceedings of the 42nd Annual Asilomar Conference on Signals, Systems and Computers, Pacific Grove, Calif, USA, October 2008.

[10] A. K. El-Sakkary, "The gap metric: robustness of stabilization of feedback systems," IEEE Transactions on Automatic Control, vol. 30, no. 3, pp. 240-247, 1985.

[11] T. T. Georgiou and M. C. Smith, "Optimal robustness in the gap metric," IEEE Transactions on Automatic Control, vol. 35, no. 6, pp. 673-686, 1990.

[12] V. R. Dehkordi and B. Boulet, "Frequency-domain robust performance condition for plant and controller uncertainty in SISO LTI systems," in Proceedings of IEEE International Conference on Computer-Aided Control Systems (CACSD '08), pp. 126-131, San Antonio, Tex, USA, September 2008.

[13] J. C. Doyle, K. Glover, P. P. Khargonekar, and B. A. Francis, Feedback Control Theory, Macmillan, New York, NY, USA, 1992.

[14] K. Zhou and J. C. Doyle, Essentials of Robust Control, PrenticeHall, Upper Saddle River, NJ, USA, 1997.

[15] J. C. Doyle, A. Packard, and K. Zhou, "Review of LFTs, LMIs, and $\mu$," in Proceedings of the 30th IEEE Conference on Decision and Control (CDC'91), pp. 1227-1232, Brighton, UK, December 1991.

[16] S. P. Boyd and L. Vandenberghe, Convex Optimization, Cambridge University Press, New York, NY, USA, 2004. 

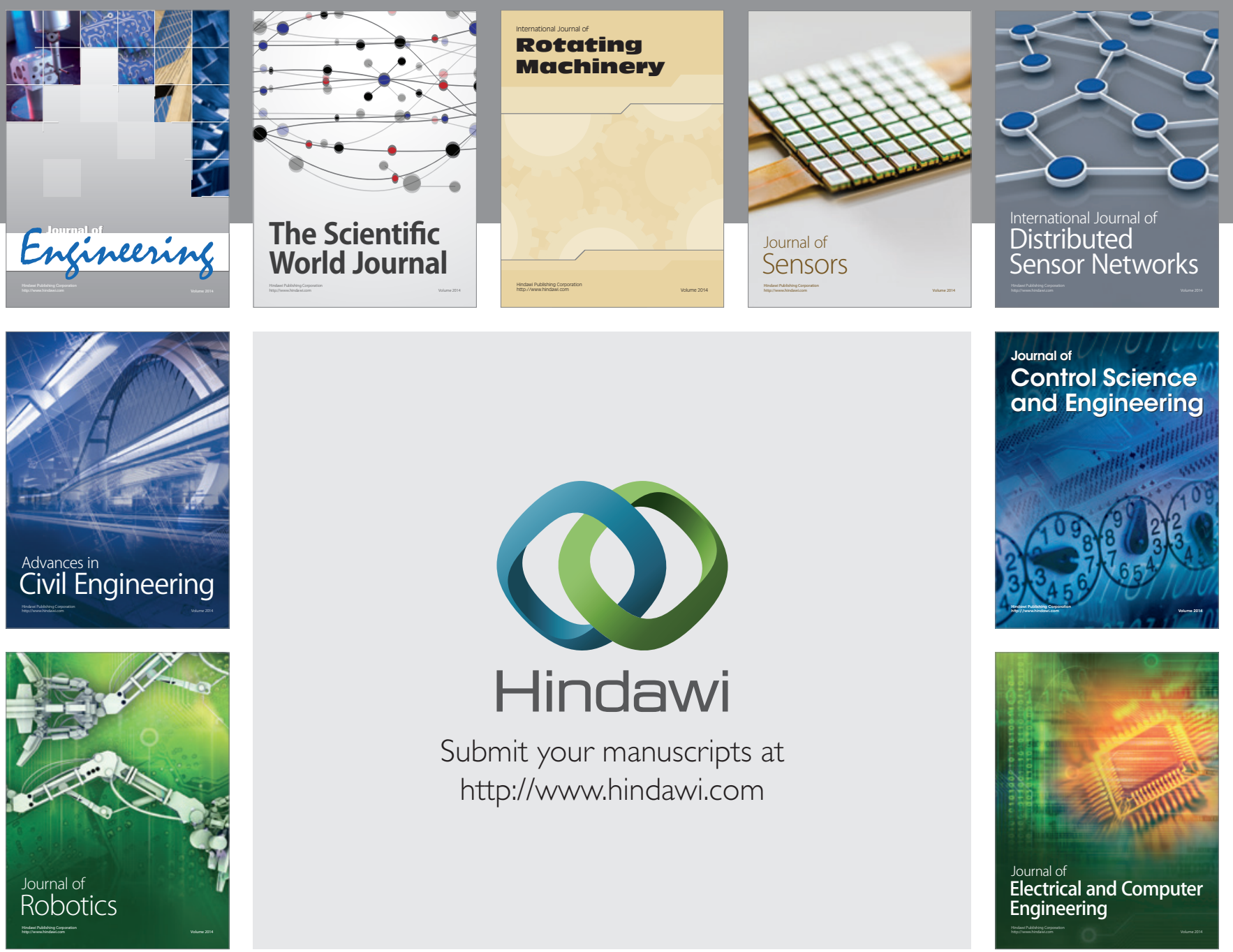

Submit your manuscripts at

http://www.hindawi.com
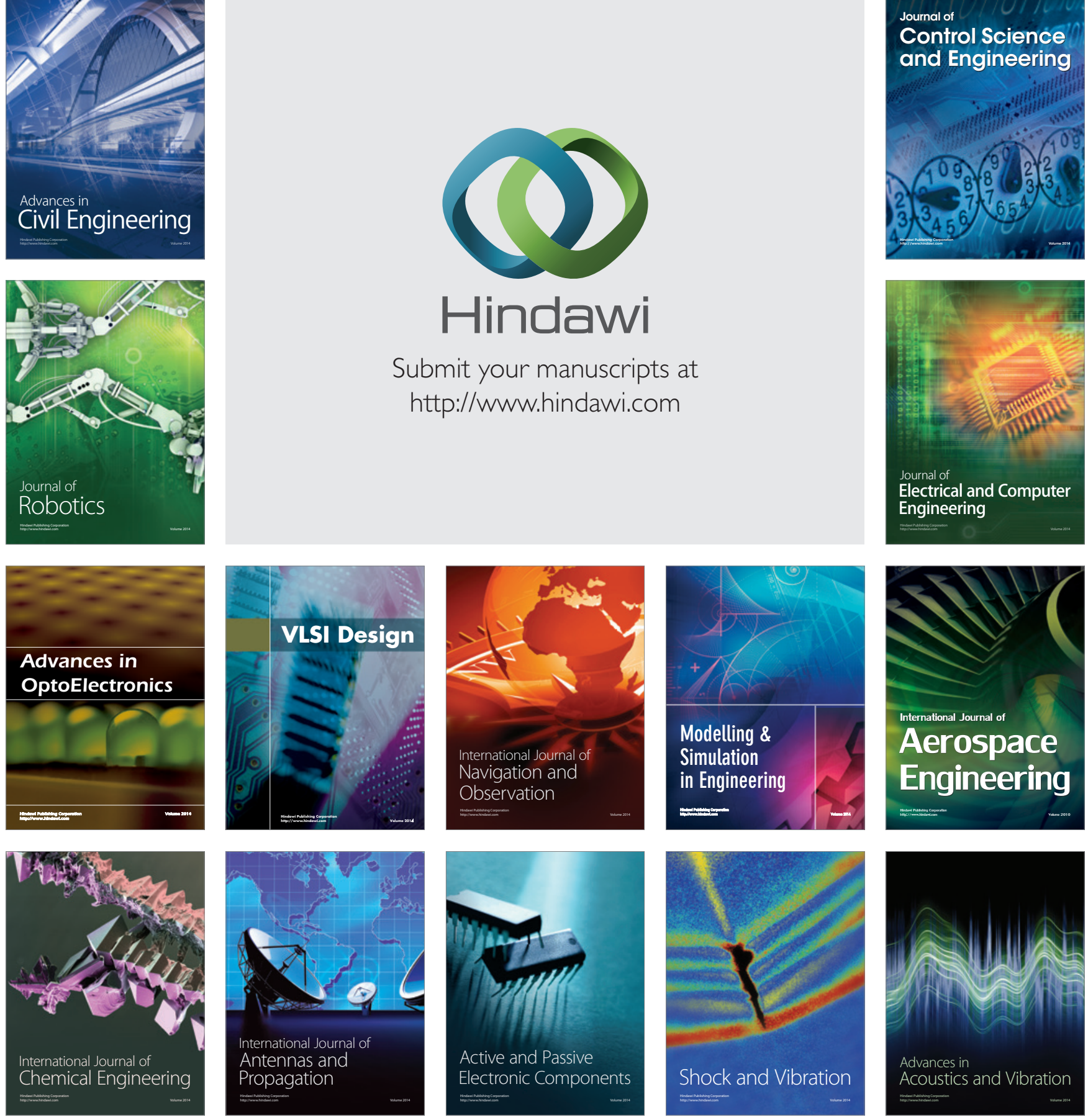